\title{
ALOCAÇÃO DE ZEROS APLICADA A SISTEMAS DE CONTROLE VIA LMI
}

\author{
Edvaldo Assunção* \\ edvaldo@dee.feis.unesp.br
}

\author{
Cristiano Quevedo Andrea* \\ cristiano@batlab.ufms.br
}

\author{
Marcelo Carvalho Minhoto Teixeira* \\ marcelo@dee.feis.unesp.br \\ ${ }^{*}$ UNESP - Universidade Estadual Paulista, \\ Departamento de Engenharia Elétrica, \\ Faculdade de Engenharia de Ilha Solteira, \\ 15385-000, Ilha Solteira, São Paulo, Brasil.
}

\begin{abstract}
A systematic procedure of zero placement to design control systems is proposed. A state feedback controller with vector gain $K$ is used to perform the pole placement. An estimator with vector gain $L$ is also designed for output feedback control. A new systematic method of zero assignment to reduce the effect of the undesirable poles of the plant and also to increase the velocity error constant is presented. The methodology places the zeros in a specific region and it is based on Linear Matrix Inequalities (LMIs) framework, which is a new approach to solve this problem. Three examples illustrate the effectiveness of the proposed method.
\end{abstract}

KEYWORDS: Zeros Placement, Control Systems, LMI.

\section{RESUMO}

Neste trabalho propõe-se um novo procedimento sistemático de alocação de zeros aplicado em sistemas de controle. Considera-se um sistema de controle com posicionamento de pólos utilizando-se realimentação de estados através de um controlador com matriz de ganhos $K$. Para realimentar a saída, projeta-se uma matriz de ganhos $L$ do estimador. É

Artigo submetido em 07/10/2005

1a. Revisão em 05/04/2006

2a. Revisão em 24/07/2006

Aceito sob recomendação do Editor Associado

Prof. José Roberto C.Piqueira proposta uma nova metodologia de alocação de zeros via inequações matriciais lineares (LMIs) para posicionar os zeros dentro de uma região de maneira a diminuir o efeito dos pólos indesejáveis, e ainda para alterar a constante de erro de velocidade do sistema. O uso de LMI para o posicionamento de zeros em uma região é inédito. Exemplos numéricos ilustram a eficiência da metodologia proposta.

PALAVRAS-CHAVE: Alocação de Zeros, Sistemas de Controle, LMI.

\section{INTRODUÇÃO}

Considerando-se a vasta literatura de controle como, por exemplo, descrito em (Ogata, 1997) e (Dorf, 2001), verificase a existência de várias metodologias para síntese de controladores. Entre estas metodologias, existe a possibilidade do projetista escolher o posicionamento dos pólos de malha fechada do sistema. Quando utiliza-se a realimentação de saída através de um compensador dinâmico ou um estimador de estados, os zeros de malha fechada são decorrentes do processo de posicionamento dos pólos. Ainda, os zeros podem ficar em algumas regiões do plano- $s$ prejudicando a dominância dos pólos. Assim, o sistema de malha fechada pode não atender as especificações para o transitório do sistema, tais como porcentagem de overshoot, tempo de subida e também o tempo de estabelecimento.

Alguns textos clássicos de sistemas de controle tais como 
(Ogata, 1997) e (Dorf, 2001) abordam os zeros de maneira muito simples, enquanto em (Franklin et al., 1994) pode-se observar que os zeros são tratados com maior importância, onde encontra-se uma metodologia que trata um processo que relaciona a posição dos zeros com a constante de erro de velocidade de um dado sistema.

Em (Moore and Bhattacharyya, 1990) utiliza-se uma técnica com o objetivo de minimizar o overshoot de um sistema. Neste contexto, utiliza-se a alocação de zeros de maneira a otimizar a percentagem de overshoot de um determinado sistema de controle. Considerando-se sistemas de rastreamento de sinais de referência, (Herjólfsson et al., 2005) propõe um método de projeto utilizando-se alocação otimizada dos zeros, no qual considera-se a entrada de referência do tipo degrau. Outro tópico de pesquisa utilizando a alocação de zeros é projetos de superfícies deslizantes aplicada em sistemas multivariáveis. Pode-se verificar em (Saeks and Murray, 1981) um método de projeto para superfícies deslizantes baseado na alocação dos zeros de transmissão da função de transferência entre o sinal de controle e a saída especificada da superfície deslizante.

Ainda pode-se verificar a alocação dos zeros em redução de modelos (Hauksdóttir, 2000), na robustez de sistemas de controle (Tu and Lin, 1992), no controle de vibração (Lee et al., 1987) entre outros temas. A formulação de projetos de sistemas de controle em termos de LMIs (Boyd et al., 1994), tem sido uma ferramenta útil para resolver um grande número de problemas (Assunção and Peres, 1999), (Teixeira et al., 2001), (Teixeira et al., 2003), (da Silva et al., 2004), (Palhares et al., 2003), (Teixeira et al., 2002), (Teixeira and Zak, 1999). As características principais das LMIs são que uma diversidade de especificações e restrições de projeto podem ser descritas na forma de LMIs, e uma vez formulado em termos de LMIs, o problema, quando existe uma solução, pode ser exatamente e eficientemente resolvido com o emprego de algoritmos de otimização convexa (Boyd et al., 1994), (Nesterov and Nemirovsky, 1994).

Em sistemas de rastreamento pode-se observar a utilidade da modificação dos zeros. Em (Assunção et al., 2004) foi apresentado um processo de otimização, baseado em LMIs, para determinar o posicionamento ótimo dos zeros para o rastreamento de sinais de controle e rejeição de distúrbios, cujo critério de desempenho são as normas $\mathcal{H}_{2}$ e $\mathcal{H}_{\infty}$ respectivamente. Neste caso os pólos podem ser arbitrariamente posicionados no plano-s.

Neste trabalho propõe-se uma metodologia para alocação dos zeros, em uma região específica do plano- $s$, de forma sistemática, com objetivo de auxiliar o desenvolvimento de projetos em sistemas de controle. O projeto de posicionamento dos zeros é descrito na forma de LMIs, que podem ser facil- mente solucionados através de algoritmos de convergência polinomial disponíveis na literatura ((Gahinet et al., 1995), (de Oliveira et al., 1997)). Pelo conhecimento dos autores, a alocação de zeros em uma região via LMI é original e inédita. Exemplos ilustram a eficiência da metodologia proposta.

\section{FORMULAÇÃO DO PROBLEMA}

Problema 1 Considere um sistema MIMO (do inglês, MultiInput Multi-Output) controlável, observável, linear e invariante no tempo:

$$
\begin{aligned}
\dot{x}(t) & =A x(t)+B u(t), \\
z(t) & =C x(t),
\end{aligned}
$$

sendo $A \in \Re^{n \times n}, B \in \Re^{n \times p}, C \in \Re^{m \times n}, x(t)$ é o vetor de estados $\left(x(t) \in \Re^{n}\right), z(t)$ é a saída de interesse $\left(z(t) \in \Re^{m}\right)$, $u(t)$ a entrada de controle $\left(u(t) \in \Re^{p}\right)$.

O objetivo do problema é projetar um controlador usando realimentação da saída tal que os pólos de malha fechada fiquem em uma determinada região especificada através de LMIs, e ainda, alocar os zeros do sistema em uma outra região especificada através de LMIs de modo a diminuir o efeito de pólos indesejáveis no desempenho do sistema.

Observação 1 O diagrama de blocos do sistema de controle utilizado neste trabalho para resolver o Problema 1 é dado na Figura 1 , sendo que $K$ é a matriz de ganho de realimentação dos estados e L é a matriz de ganho do estimador de estados utilizado na realimentação da saída. Utiliza-se a matriz $M \in \Re^{n \times p}$ e a matriz $N \in \Re^{p \times p}$ para solucionar o problema da alocação dos zeros.

Para o projeto do controlador $K$ ilustrado na Figura 1 utilizase uma metodologia já consagrada na literatura de sistemas de controle. Desta maneira o controlador $K$ pode ser obtido através do Teorema 1 descrito a seguir.

Teorema 1 Considere o sistema (1) com o controlador de realimentação de estados, dado por $u(t)=-K x(t)$, e ainda que os parâmetros $\rho, q, \alpha$ e $\theta$ são fixos. Se existe solução para as LMIs descritas em (2) então pode-se obter o controlador $K$ que estabiliza o sistema (1) com alocação de pólos na região mostrada pela Figura 2.

$$
\begin{aligned}
& A P+P A^{\prime}-B Y-Y^{\prime} B^{\prime}+2 \alpha P<0, \\
& {\left[\begin{array}{cc}
-\rho P & A P-B Y+q P \\
P A^{\prime}-Y^{\prime} B^{\prime}+q P & -\rho P
\end{array}\right]<0,} \\
& {\left[\begin{array}{cc}
\operatorname{sen} \theta(T 1) & \cos \theta\left(T_{2}\right) \\
\cos \theta\left(T_{2}^{\prime}\right) & \operatorname{sen} \theta\left(T_{1}\right)
\end{array}\right]<0,} \\
& P>0 \text {, }
\end{aligned}
$$




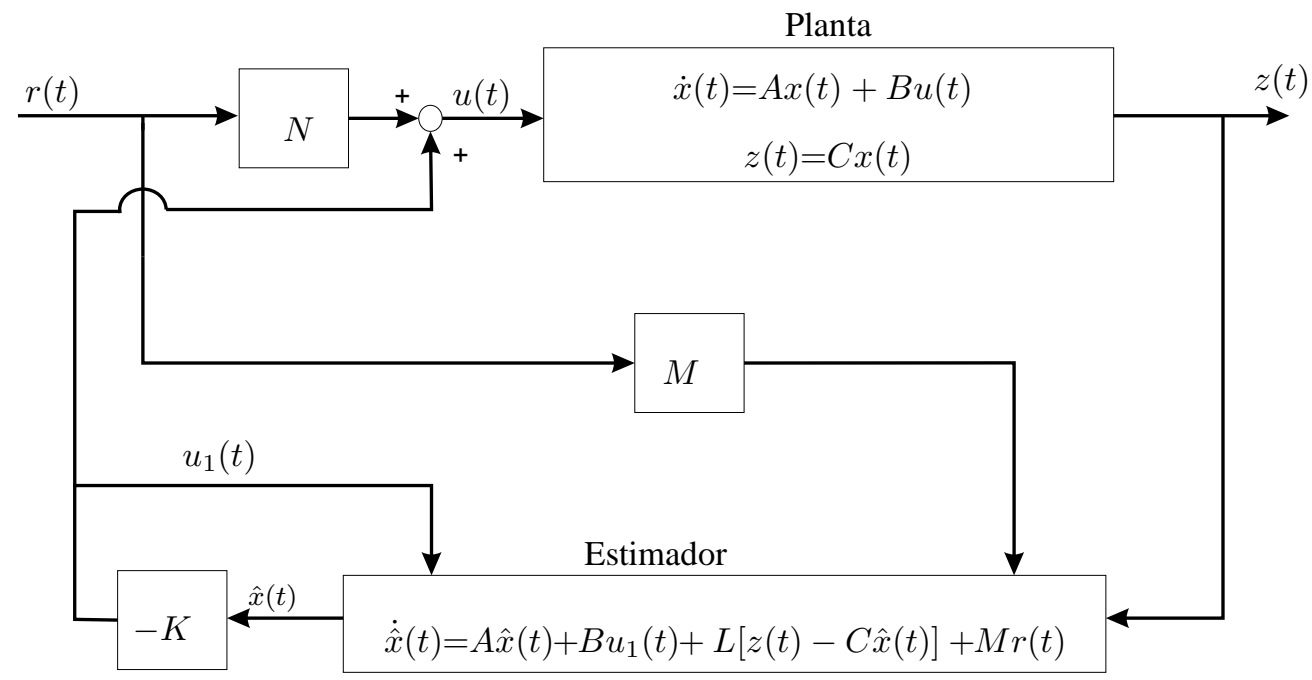

Figura 1: Sistema de controle com alocação de zeros.

sendo

$$
\begin{aligned}
& T_{1}=A P-B Y+P A^{\prime}-Y^{\prime} B^{\prime}, \\
& T_{2}=A P-B Y-P A^{\prime}+Y^{\prime} B^{\prime},
\end{aligned}
$$

e $Y \in \Re^{p \times n}, P \in \Re^{n \times n}$ e $P=P^{\prime}$. Quando as LMIs acima são factíveis, um controlador $K$ que satisfaz o problema é $K=Y P^{-1}$, sendo $P$ e $Y$ soluções factíveis de (2).

Prova: vide (Chilali and Gahinet, 1996).

Para a solução do Problema 1, pode-se utilizar o Teorema 1 para determinar o controlador de realimentação de estados para o sistema (1). Posteriormente, projeta-se a matriz de ganho do estimador de estados $L$ através do Teorema 2. O estimador de estados é utilizado na realimentação da saída $z(t)$, como descrito na Figura 1. Neste processo considerase a matriz $M$ de alocação de zeros ilustrado na Figura 1 nulo.

Teorema 2 Considera-se o sistema (1) com estimador de estados ilustrado na Figura 1, e ainda que o parâmetro $\rho_{L}$ e $q_{L}$ são fixos. Se existe solução para as LMIs descritas em (3) então pode-se obter a matriz de ganhos $L$ do estimador de estados com alocação de pólos em uma região limitada por um círculo com raio $\rho_{L}$ e centro em $\left(-q_{L}, 0\right)$ ilustrada na Figura 2.

$$
\begin{aligned}
& A^{\prime} \check{P}+\check{P} A-W C-C^{\prime} W^{\prime}<0, \\
& {\left[\begin{array}{cc}
-\rho_{L} \check{P} & q_{L} \check{P}+\check{P} A-W C \\
\check{P} q_{L}+A^{\prime} \check{P}-C^{\prime} W^{\prime} & -\rho_{L} \check{P}
\end{array}\right]<0,} \\
& \check{P}>0 \text {, }
\end{aligned}
$$

$\check{P} \in \Re^{n \times n}$ e $W \in \Re^{n \times m}$ são soluções factíveis de (3) e $\check{P}=\check{P}^{\prime}$. Uma matriz de ganhos $L$ do estimador que satisfaz o problema é $L=\check{P}^{-1} W$.

Prova: considera-se o sistema descrito na forma de espaço de estado em (1) e o estimador conforme ilustrado na Figura 1 dado por:

$$
\begin{gathered}
\dot{\hat{x}}(t)=A \hat{x}(t)+B u_{1}(t)+L[z(t)-C \hat{x}(t)], \quad e \\
u_{1}(t)=-K \hat{x}(t) .
\end{gathered}
$$

Para obter a equação de erro do estimador, subtrai-se $\dot{x}(t)$ por $\dot{\hat{x}}(t)$. Neste contexto considera-se a entrada de referência $r(t)$ nula, e desta maneira tem-se $u(t)=u_{1}(t)$ :

$$
\dot{x}(t)-\dot{\hat{x}}(t)=A x(t)-A \hat{x}(t)-L[C x(t)-C \hat{x}(t)],
$$

então,

$$
\dot{x}(t)-\dot{\hat{x}}(t)=(A-L C)[x(t)-\hat{x}(t)] .
$$

Definindo-se o vetor erro e $(t)$ como a diferença entre $x(t) e$ $\hat{x}(t)$ tem-se:

$$
\dot{e}(t)=(A-L C) e(t) .
$$

Desta maneira, se a matriz $(A-L C)$ tiver autovalores estáveis, o vetor erro convergirá para zero seja qual for o valor inicial do vetor erro e(0), vide (Ogata, 1997) para maiores detalhes. A matriz $(A-L C)$ tem autovalores estáveis se e somente se existir uma matriz $P_{1}>0$ tal que:

$$
(A-L C)^{\prime} P_{1}+P_{1}(A-L C)<0 .
$$




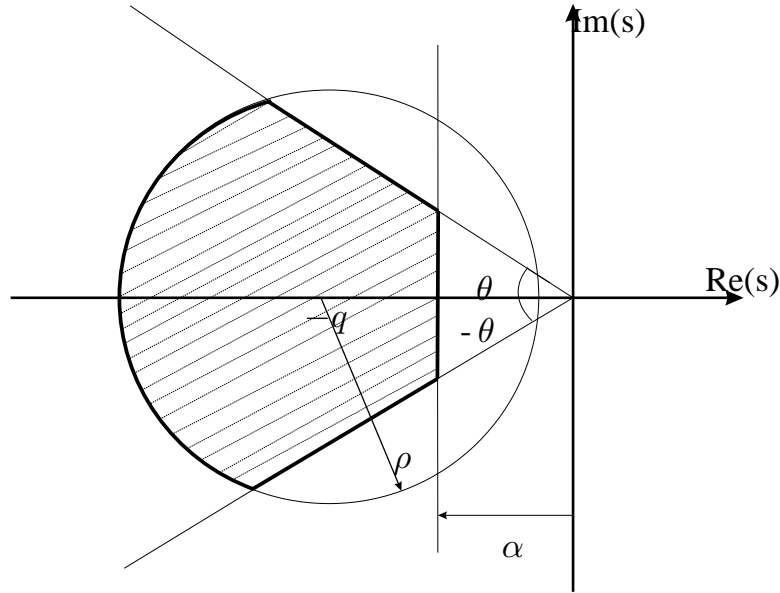

Figura 2: Projeto do controlador com matriz de ganhos $K$ utilizado na realimentação dos estados estimados da planta. A área hachurada representa a região para alocação dos pólos.

Ainda, aloca-se os autovalores da matriz $(A-L C)$ uma região limitada por uma circunferência de raio $\rho_{L}$ com centro em $\left(-q_{L}, 0\right)$ se existir uma matriz $P_{2}>0$ tal que (Chilali and Gahinet, 1996):

$$
\left[\begin{array}{cc}
-\rho_{L} P_{2} & q_{L} P_{2}+P_{2}(A-L C) \\
P_{2} q_{L}+(A-L C)^{\prime} P_{2} & -\rho_{L} P_{2}
\end{array}\right]<0 .
$$

Em 7, os parâmetros $\rho_{L}$ e $q_{L}$ são fixos e especificados pelo projetista. Realizando-se manipulações algébricas em (6) $e$ (7), com $P_{1}=P_{2}=\check{P}$ e $W=P L$ obtem-se as LMIs descritas no Teorema 2.

Para a resolução completa do Problema 1, aloca-se os zeros através dos parâmetros $M$ e $N$ ilustrados na Figura 1 com o objetivo de atenuar os efeitos de pólos indesejáveis ao desempenho do sistema, e ainda pode-se utilizar o posicionamento dos zeros para alterar a constante de erro de velocidade em sistemas de controle. A equação dos zeros do sistema da Figura 1, considerando-se como entrada $r(t)$ e saída $u(t)$ é descrita no Teorema 3.

Teorema 3 Considere $K, L, M$ e $N$ fixos, se existe solução para a equação (8) então pode-se determinar os zeros da função de transferência de $R(s)$ para $U(s)$ do sistema ilustrado na Figura 1.

$$
\operatorname{det}\left(s I-A_{z}\right)=0,
$$

sendo $A_{z}=A-B K-L C+M N^{-1} K$.

Prova: (Franklin et al., 1994) Aplica-se uma entrada $r(t)$ na forma exponencial ao sistema (1),

$$
r(t)=r_{o} e^{s t},
$$

sendo que $s=z_{i}$, e $z_{i}$ é um zero de $r(t)$ para $u(t)$. Logo,

$$
r(t)=r_{o} e^{z_{i} t}
$$

Neste caso, a saída $z(t)$ é identicamente igual a zero, pois se $z_{i}$ é um zero de transmissão de $r(t)$ para $u(t)$, então necessariamente $z_{i}$ é um zero de transmissão de $r(t)$ para $z(t)$ a menos que ocorra cancelamento de pólos e zeros. Considerando-se que as condições iniciais do sistema são nulas, tem-se (Franklin et al., 1994):

$$
z(t)=0, \text { se } r(t)=r_{o} e^{z_{i} t} .
$$

Desta modo, o estado estimado $\hat{x}(t)$ pode ser descrito da seguinte maneira:

$$
\hat{x}(t)=\hat{x}_{o} e^{z_{i} t} .
$$

Derivando-se (9), tem-se:

$$
\dot{\hat{x}}(t)=z_{i} \hat{x}_{o} e^{z_{i} t},
$$

mas,

$$
\dot{\hat{x}}(t)=(A-B K-L C) \hat{x}_{o} e^{z_{i} t}+M r_{o} e^{z_{i} t},
$$

então, igualando-se (10) a (11), obtem-se

$$
\begin{array}{r}
z_{i} \hat{x}_{o} e^{z_{i} t}=(A-B K-L C) \hat{x}_{o} e^{z_{i} t}+M r_{o} e^{z_{i} t}, \\
z_{i} \hat{x}_{o} e^{z_{i} t}-(A-B K-L C) \hat{x}_{o} e^{z_{i} t}-M r_{o} e^{z_{i} t}=0,
\end{array}
$$

$o u$,

$$
\left[\begin{array}{ll}
z_{i}-A+B K+L C & -M
\end{array}\right]\left[\begin{array}{l}
\hat{x}_{o} \\
r_{o}
\end{array}\right]=0 .
$$

Ainda, tem-se que a entrada a entrada $r(t)=r_{o} e^{z_{i} t}$ torna $u(t)=0$, pois $z_{i}$ é um zero do sistema. Desta maneira, de acordo com a Figura 1, pode-se descrever $u(t)$ da seguinte maneira:

$$
\begin{aligned}
& u(t)=-K \hat{x}(t)+N r(t), \\
& u(t)=-K \hat{x}_{o} e^{z_{i} t}+N r_{o} e^{z_{i} t},
\end{aligned}
$$

então,

$$
-K \hat{x}_{o} e^{z_{i} t}+N r_{o} e^{z_{i} t}=0,
$$

ou,

$$
\left[\begin{array}{ll}
-K & N
\end{array}\right]\left[\begin{array}{l}
\hat{x}_{o} \\
r_{o}
\end{array}\right]=0
$$

Reagrupando-se (12) e (13) tem-se:

$$
\left[\begin{array}{cc}
z_{i}-A+B K+L C & -M \\
-K & N
\end{array}\right]\left[\begin{array}{l}
\hat{x}_{o} \\
r_{o}
\end{array}\right]=\left[\begin{array}{l}
0 \\
0
\end{array}\right]
$$


Em (14), $z_{i}$ é um zero de $r(t)$ para $u(t)$ do sistema ilustrado na Figura 1 e (14) tem solução não trivial. A solução para (14) é equivalente a solucionar:

$$
\operatorname{det}\left[\begin{array}{cc}
z_{i}-A+B K+L C & -M \\
-K & N
\end{array}\right]=0 .
$$

Dividindo-se a última coluna por uma matriz não nula $N$ e então adicionando-se a primeira coluna o produto de K pela última coluna, tem-se:

$$
\operatorname{det}\left[\begin{array}{cc}
z_{i}-A+B K+L C-M N^{-1} K & -M N^{-1} \\
0 & 1
\end{array}\right]=0,
$$

ou ainda, considerando-se $z_{i}=s$,

$$
\operatorname{det}\left(s I-A+B K+L C-M N^{-1} K\right)=0 .
$$

Observa-se que o Teorema 3 apresenta a equação dos zeros de $r(t)$ para $u(t)$ ilustrados na Figura 1, e neste trabalho são propostas metodologias de alocação de zeros descritas nos Teoremas 4, 5 e 6 . Os métodos de alocação de zeros propostos possibilitam o posicionamento dos zeros da função de transferência de $R(s)$ para $U(s)$, isto é, permitem o posicionamento dos zeros de $r(t)$ para $u(t)$.

Teorema 4 Considere os parâmetros $K, L, \rho_{z}$ e $q_{z}$ fixos. Se existe solução para a LMI descrita em (15) então pode-se alocar os zeros da função de transferência de $R(s)$ para $U(s)$ (Figura 1) em uma região limitada por um círculo de raio $\rho_{z}$ com centro em $\left(-q_{z}, 0\right)$ ilustrada na Figura 2.

$$
\left[\begin{array}{cc}
-\rho_{z} Q & \Omega \\
\Omega^{\prime} & -\rho_{z} Q
\end{array}\right]<0,
$$

sendo $\Omega=q_{z} Q+Q A-Q B K-Q L C+F K$ e $F=$ $Q M N^{-1} \in \Re^{n \times p}$.

Prova: segundo o Teorema 1, mas especificamente em (Chilali and Gahinet, 1996), para que os autovalores de uma dada matriz Ã estejam alocados em uma região do plano-s limitados por uma circunferência de raio $\rho_{z}$ com centro em $\left(-q_{z}, 0\right)$, deve existir uma matriz $Q \in \Re^{n \times n}, Q=Q^{\prime}>0$, tal que a seguinte equação deve seja satisfeita:

$$
\left[\begin{array}{cc}
-\rho_{z} Q & q_{z} Q+Q \tilde{A} \\
q_{z} Q+\tilde{A}^{\prime} Q & -\rho_{z} Q
\end{array}\right]<0
$$

Então, substituindo-se a matriz Ã pela matriz $A_{z}$ (indicada em (8)) na equação (16), obtem-se a equação (15), e aloca-se os autovalores de $A_{z}$ na região limitada pela circunferência, e conseqüentemente, segundo o Teorema 3, aloca-se os zeros nesta região, pois os autovalores de $A_{z}$ são os zeros de $r(t)$ para $u(t)$.
Teorema 5 Considere os parâmetros $K, L \mathrm{e}-\alpha_{z}$ fixos. Se existe solução para a LMI descrita em (17) então pode-se alocar os zeros de $R(s)$ para $U(s)$ (Figura 1) em uma região limitada por uma reta vertical em $-\alpha_{z}$ ilustrada na Figura 2.

$$
\begin{gathered}
\check{Q} A-\check{Q} B K-\check{Q} L C+\check{F} K+A^{\prime} \check{Q}-K^{\prime} B^{\prime} \check{Q} \\
-C^{\prime} L^{\prime} \check{Q}+K^{\prime} \check{F}^{\prime}+2 \alpha_{z} \check{Q}<0,
\end{gathered}
$$

sendo $\check{F}=\check{Q} M N^{-1} \in \Re^{n \times p}$.

Prova: para que os autovalores da matriz Ã estejam alocados em uma região do plano-s limitados por uma reta vertical em $-\alpha$ (Figura 2), deve existir uma matriz $\check{Q} \in \Re^{n \times n}$ positiva definida tal que a equação seguinte seja satisfeita (Boyd et al., 1994):

$$
\tilde{A}^{\prime} \check{Q}+\check{Q} \tilde{A}+2 \alpha_{z} \check{Q}<0 .
$$

Então, substituindo-se a matriz $\tilde{A}$ pela matriz $A_{z}$ em (18) obtem-se a equação (17), e aloca-se os autovalores de $A_{z}$ na região limitada por uma reta vertical em $-\alpha_{z}$, e conseqüentemente aloca-se os zeros nesta região, pois os autovalores de $A_{z}$ são os zeros de $r(t)$ para $u(t)$.

Teorema 6 Considere os parâmetros $K, L$ e $\theta$ fixos. Se existir solução para a LMI descrita em (19) então pode-se alocar os zeros de $R(s)$ para $U(s)$ (Figura 1) em uma região limitada por duas semi-retas de ângulos $\theta \mathrm{e}-\theta$ ilustrada na Figura 2.

$$
\left[\begin{array}{cc}
\left(\operatorname{sen} \theta_{z}\right) R_{\theta} & \left(\cos \theta_{z}\right) T_{\theta} \\
\left(\cos \theta_{z}\right) T_{\theta}^{\prime} & \left(\operatorname{sen} \theta_{z}\right) R_{\theta}
\end{array}\right]<0,
$$

sendo,

$$
\begin{aligned}
R_{\theta}= & A^{\prime} \bar{Q}-K^{\prime} B^{\prime} \bar{Q}-C^{\prime} L^{\prime} \bar{Q}+K^{\prime} \bar{F}^{\prime}+\bar{Q} A \\
& -\bar{Q} B K-\bar{Q} L C+\bar{F} K, \\
T_{\theta}= & \bar{Q} A-\bar{Q} B K-\bar{Q} L C+\bar{F} K-A^{\prime} \bar{Q} \\
& +K^{\prime} B^{\prime} \bar{Q}+C^{\prime} L^{\prime} \bar{Q}-K^{\prime} \bar{F}^{\prime},
\end{aligned}
$$

sendo $\bar{F}=\bar{Q} M N^{-1} \in \Re^{n \times p}$.

Prova: segundo o Teorema 1, mais especificamente em (Chilali and Gahinet, 1996), para que os autovalores da matriz $\tilde{A}$ estejam alocados em uma região do plano-s limitada por duas semi-retas de ângulo $\theta_{z} e-\theta_{z}$, deve existir uma matriz $\bar{Q} \in \Re^{n \times n}, \bar{Q}=\bar{Q}^{\prime}>0$, tal que a seguinte equação seja satisfeita:

$$
\left[\begin{array}{cc}
\operatorname{sen} \theta_{z}\left(\bar{Q} \tilde{A}+\tilde{A}^{\prime} \bar{Q}\right) & \cos \theta_{z}\left(\bar{Q} \tilde{A}-\tilde{A}^{\prime} \bar{Q}\right) \\
\cos \theta_{z}\left(\tilde{A}^{\prime} \bar{Q}-\bar{Q} \tilde{A}\right) & \operatorname{sen} \theta_{z}\left(\bar{Q} \tilde{A}+\tilde{A}^{\prime} \bar{Q}\right)
\end{array}\right]<0 .
$$

Então, substituindo-se a matriz $\tilde{A}$ pela matriz $A_{z}$ em (20) obtem-se a equação (19), e aloca-se os autovalores de $A_{z}$ 
na região limitada pelas semi-retas com ângulos $\theta_{z} e-\theta_{z}$, e conseqüentemente aloca-se os zeros nesta região, pois os autovalores de $A_{z}$ são os zeros de $r(t)$ para $u(t)$.

Observa-se que os Teoremas 4, 5 e 6 não são conjuntamente convexos, pois podem admitir diferentes soluções $(Q \neq \check{Q} \neq$ $\bar{Q})$. Então é proposto o Corolário 1 para o projeto de alocação de zeros na região ilustrada na Figura 2, para solução do problema 1.

Corolário 1 Considere os parâmetros $K, L, \alpha_{z}, \rho_{z}, q_{z}$ e $\theta_{z}$ fixos. Se existir solução para as LMIs descritas em (21) então pode-se alocar os zeros de $R(s)$ para $U(s)$ (Figura 1) na região mostrada pela Figura 2 .

$$
\begin{array}{r}
R_{\alpha}+R_{\alpha}^{\prime}+2 \alpha_{z} X<0, \\
{\left[\begin{array}{cc}
-\rho_{z} X & R_{\theta} \\
R_{\theta}^{\prime} & -\rho_{z} X
\end{array}\right]<0} \\
{\left[\begin{array}{cc}
\left(\operatorname{sen} \theta_{z}\right) R_{x} & \left(\cos \theta_{z}\right) T_{x} \\
\left(\cos \theta_{z}\right) T_{x}^{\prime} & \left(\operatorname{sen} \theta_{z}\right) R_{x}
\end{array}\right]<0,} \\
X>0,
\end{array}
$$

sendo

$$
\begin{aligned}
R_{\alpha}= & X A-X B K-X L C+F K+A^{\prime} X \\
& -K^{\prime} B^{\prime} X-C^{\prime} L^{\prime} X+K^{\prime} F^{\prime}, \\
R_{\theta}= & q_{z} X+X A-X B K-X L C+F K, \\
R_{x}= & A^{\prime} X-K^{\prime} B^{\prime} X-C^{\prime} L^{\prime} X+K^{\prime} F^{\prime} \\
& +X A-X B K-X L C+F K, \\
T_{x}= & X A-X B K-X L C+F K-A^{\prime} X \\
& +K^{\prime} B^{\prime} X+C^{\prime} L^{\prime} X-K^{\prime} F^{\prime},
\end{aligned}
$$

As matrizes $X \in \Re^{n \times n}$ e $F \in \Re^{n \times p}$ são soluções factíveis de (21). Os parâmetros para alocação de zeros são determinados por:

$$
M N^{-1}=X^{-1} F=J .
$$

Prova: Com o objetivo de impor convexidade conjunta para as regiões LMIs dos Teoremas 4, 5 e 6, isto é, obter uma solução comum, faz-se:

$$
Q=\check{Q}=\bar{Q}=X .
$$

Adotando-se $F=X M N^{-1}$ e agrupando todas a LMIs dos Teoremas 4, 5 e 6, obtêm-se o projeto na forma de LMIs que permite a alocação de zeros na região do plano-s mostrada na Figura 2.

Desta maneira através do Colorário 1 pode-se alocar os zeros de $r(t)$ para $u(t)$ ilustrados na Figura 1 na região mostrada pela Figura 2. Contudo, quando a região para a alocação dos zeros for muito restritiva, pode não existir uma solução factível para o problema. Então, cabe ao projetista estipular uma área adequada e coerente para a alocação de zeros de modo que o problema seja solucionado.

Observação 2 Neste trabalho utiliza-se um estimador de estados no processo de realimentação do sistema, desta maneira, pode-se alocar $2 n$ pólos e ainda $n$ zeros. Neste processo aloca-se os zeros de $R(s)$ para $U(s)$, entretanto não altera-se o posicionamento dos zeros da planta, caso existam.

Analisando-se a Figura 1 pode-se determinar a função de transferência $R(s)$ para $Z(s)$ de acordo com a equação seguinte:

$$
\frac{Z(s)}{R(s)}=C_{m}\left(s I-A_{m}\right)^{-1} B_{m}
$$

sendo,

$$
\begin{aligned}
A_{m}= & {\left[\begin{array}{cc}
A & -B K \\
L C & A-B K-L C
\end{array}\right], B_{m}=\left[\begin{array}{c}
B N \\
M
\end{array}\right], } \\
& \text { e } C_{m}=\left[\begin{array}{ll}
C & 0
\end{array}\right] .
\end{aligned}
$$

Pode-se ainda utilizar a metodologia de alocação de zeros para atingir ganho unitário para uma entrada do tipo degrau, utilizando-se o Teorema 7.

Teorema 7 Considera-se a matriz $N$ ilustrado na Figura 1. Se existir solução para a equação (25), então pode-se obter a matriz $N$ que proporciona ganho unitário para o sistema considerando-se uma entrada do tipo degrau:

$$
N=-\left(G_{1} B+G_{2} J\right)^{-1}
$$

sendo $\left[\begin{array}{ll}G_{1} & G_{2}\end{array}\right]=C_{m}\left(A_{m}\right)^{-1}, G_{1} \in \Re^{m \times n}$ e $G_{2} \in \Re^{m \times n}$.

Prova: Considerando-se ganho unitário em regime permanente para uma entrada do tipo degrau, tem-se em (24),

$$
I=C_{m}\left(-A_{m}\right)^{-1} B_{m}
$$

expandindo $B_{m}$ em (26), obtem-se:

$$
I=\left[\begin{array}{ll}
G_{1} & G_{2}
\end{array}\right]\left[\begin{array}{c}
B N \\
M
\end{array}\right] .
$$

Note que $A_{m}^{-1}$ existe devido ao fato dos pólos do sistema controlado estarem no lado esquerdo do plano-s. De (22), temse $M N^{-1}=X^{-1} F=J$. Substituindo-se $M=J N$ em (27), pode-se obter a matriz $N$ de correção de erro de regime para entrada do tipo degrau descrito na equação (25). 
Este processo de obtenção dos parâmetros de alocação dos zeros pode ser não robusto, devido a variações na planta. Porém como a metodologia de projeto para alocação de zeros é descrita na forma de LMIs, pode-se incluir incertezas paramétricas da planta ao projeto, tornando-o robusto. Isto é tópico futuro de pesquisas por parte dos autores.

Esta metodologia para alocação de zeros pode ser utilizada em projetos de sistemas de controle com o objetivo de atenuar os efeitos indesejáveis de determinados pólos presentes no sistema, e ainda alterar a constante de erro de velocidade de um dado sistema.

\section{EXEMPLOS NUMÉRICOS}

\subsection{Exemplo 1}

Considera-se um sistema linear invariante no tempo de terceira ordem que representa a dinâmica do ângulo de rolamento de um avião a jato de alto desempenho, vide (Dorf, 2001). Seja o sistema descrito na forma de variáveis de estado e dado por:

$$
\begin{aligned}
{\left[\begin{array}{l}
\dot{x}_{1}(t) \\
\dot{x}_{2}(t) \\
\dot{x}_{3}(t)
\end{array}\right] } & =\left[\begin{array}{ccc}
-14 & -28 & -48 \\
1 & 0 & 0 \\
0 & 1 & 0
\end{array}\right]\left[\begin{array}{l}
x_{1}(t) \\
x_{2}(t) \\
x_{3}(t)
\end{array}\right]+\left[\begin{array}{l}
1 \\
0 \\
0
\end{array}\right] u(t), \\
z(t) & =\left[\begin{array}{lll}
0 & 0 & 5
\end{array}\right]\left[\begin{array}{l}
x_{1}(t) \\
x_{2}(t) \\
x_{3}(t)
\end{array}\right],
\end{aligned}
$$

sendo $x(t)$ o vetor de estados, $u(t)$ o sinal de controle e $z(t)$ a saída do sistema.

Para o projeto do controlador $K$ utiliza-se a metodologia indicada no Teorema 1 e adota-se uma região para alocação dos pólos limitada de acordo com a Figura 2, sendo que os parâmetros de posicionamento dos pólos são: uma circunferência de raio $\rho=8$ com centro em $(0,0)$, duas semi-retas com ângulo $\theta<50^{\circ}$ e uma região limitada a esquerda da reta vertical em $\alpha=-5$.

Utiliza-se a metodologia apresentada no Teorema 2 para a determinação da matriz de ganhos $L$ do estimador de estados. Adota-se uma região para o posicionamento dos pólos do estimador limitada por uma circunferência de raio $\rho_{L}=3$ com centro em $(-10,0)$. O controlador $K$ e a matriz de ganhos $L$ do estimador obtidos são apresentados a seguir:

$$
K=\left[\begin{array}{lll}
2,700 & 86,845 & 246,323
\end{array}\right] \text { e } L=\left[\begin{array}{c}
-30,155 \\
8,519 \\
3,089
\end{array}\right] \text {. }
$$

O mapeamento de pólos e zeros para o sistema é ilustrado na Figura 3. Neste processo, o posicionamento dos zeros são decorrentes da imposição dos pólos.

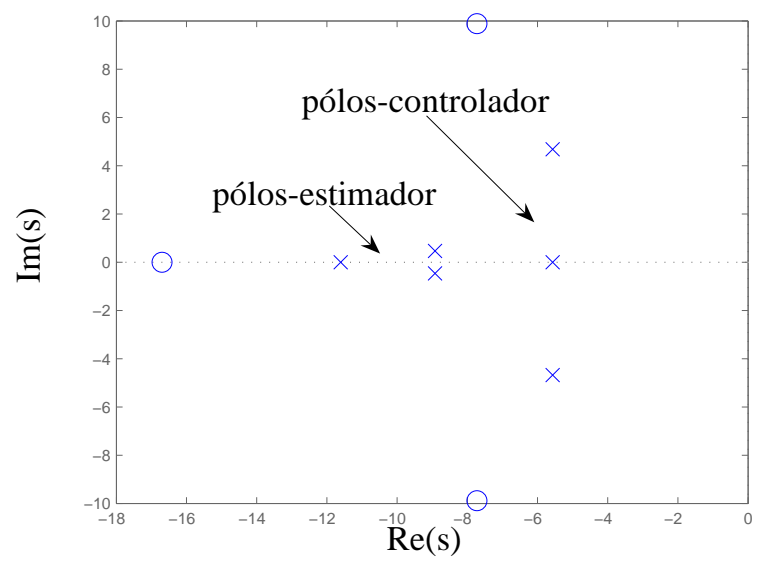

Figura 3: Mapeamento de pólos e zeros do sistema com observador e controlador.

Na Figura 4 é ilustrada a resposta do sistema projetado sem alocação de zeros, considerando-se uma entrada do tipo degrau unitário. Observa-se na Figura 4(b) que o sinal de saída do sistema apresenta uma influência relativa aos pólos do estimador, pois o tempo de estabelecimento é 1,51 segundos, enquanto que no projeto inicial apenas com o controlador $K$ o tempo de estabelecimento é 1,03 segundos, como pode-se verificar na Figura 4(a).

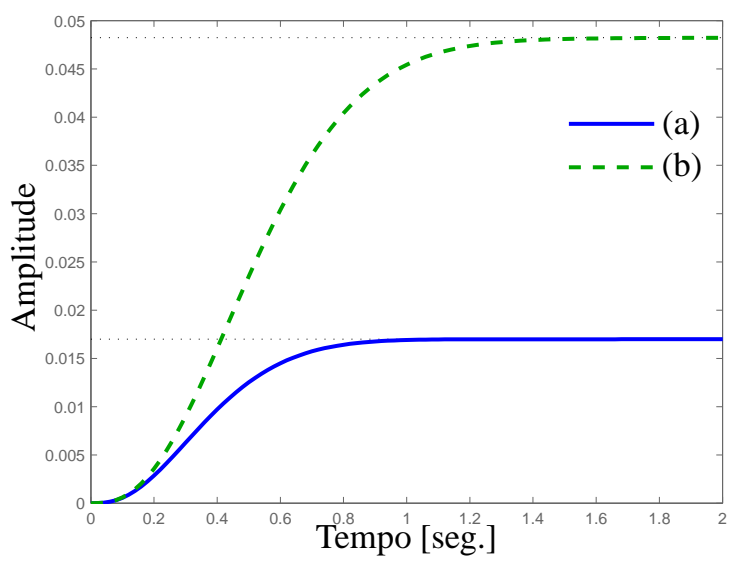

Figura 4: Resposta a entrada de um degrau unitário. (a) para o projeto somente com o controlador, (b) para o projeto com o estimador e o controlador.

Utiliza-se a metodologia para alocação de zeros descrita no Teorema 1 com objetivo de atenuar o efeito dos pólos do estimador no sistema. Então, aloca-se os zeros próximos aos pólos do estimador. Para o processo de alocação de zeros, 


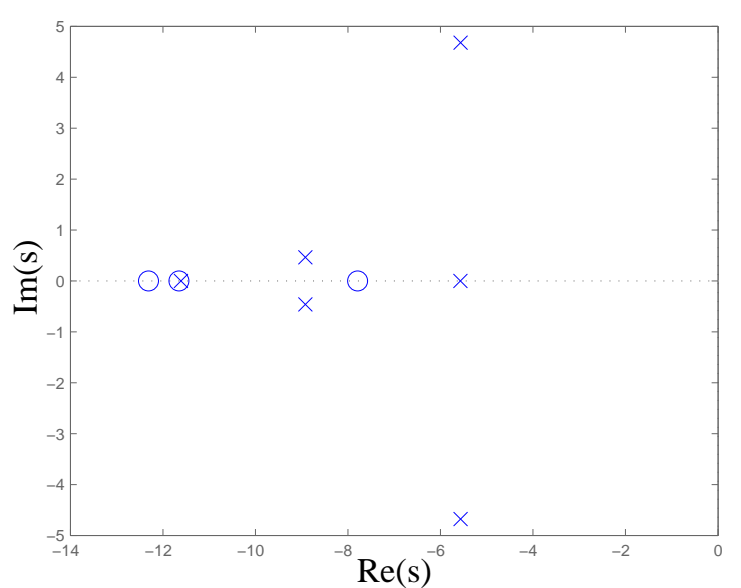

Figura 5: Mapeamento de pólos e zeros do sistema com controlador, observador e após alocação dos zeros.

adota-se a região de acordo com a Figura 2 limitada por uma circunferência de raio $\rho_{z}=3$ com centro em $(-10,0)$ do plano- $s$. Ainda, inclui-se 2 semi-retas com ângulo $\theta_{z}<30^{\circ}$ ao conjunto de restrições para a alocação dos zeros e um plano a esquerda da reta vertical em $\alpha_{z}=-7,1$.

Após realizar-se a alocação dos zeros utilizando o Teorema 1, utiliza-se o Teorema 7 para obter a matriz $N$ e posteriormente obtem-se a matriz $M$. Observa-se no mapeamento de pólos e zeros ilustrado na Figura 5 que os zeros foram posicionados próximos aos pólos do estimador, e conseqüentemente diminuindo o efeito destes pólos no sistema.

Os parâmetros de alocação de zeros $M$ e $N$ obtidos são:

$$
M=\left[\begin{array}{c}
51,75 \\
-0,63 \\
-0,08
\end{array}\right] \text { e } N=50,22
$$

Os pólos de malha fechada do sistema são: $-5,566 \pm 4,677 j$; $-5,567$ relativos ao controlador e $-11,609 ;-8,919 \pm$ $0,463 j$ relativos ao estimador. Após a alocação, os zeros do sistema são: $-9,64 \pm 0,719 j ;-11,61$.

Na Figura 6 é ilustrada a resposta do sistema para uma entrada do tipo degrau unitário após a alocação dos zeros. Analisando-se a Figura 6 constata-se que a resposta do sistema apresenta características relacionadas com os pólos do controlador. Este fator mostra a diminuição do efeito dos pólos do estimador ao desempenho global do sistema.

Neste exemplo utilizou-se a alocação de zeros para um projeto em sistemas de controle com o objetivo de atenuar o efeito de pólos indesejáveis. Observa-se que apesar dos pólos do estimador não estarem posicionados cinco vezes distantes dos pólos do controlador, como é sugerido em pro-

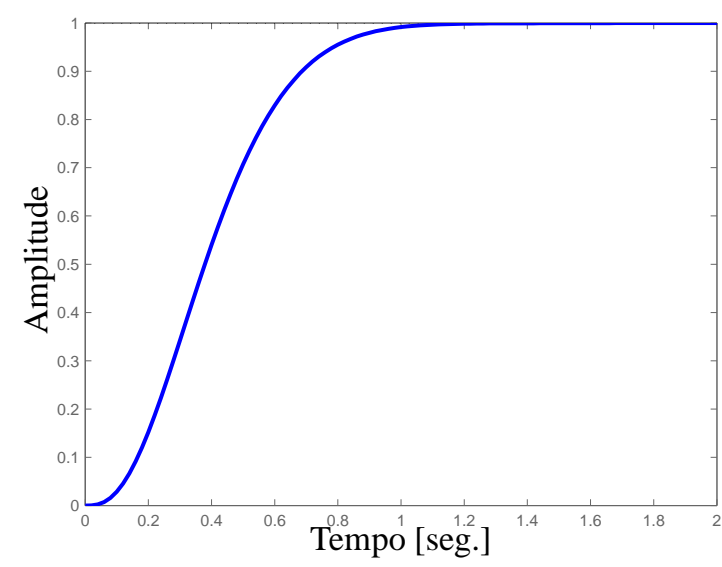

Figura 6: Resposta a entrada de um degrau unitário com controlador, observador e após alocação dos zeros.

jetos de estimadores por algumas literaturas, por exemplo (Ogata, 1997), o sistema apresenta o desempenho relativo somente aos pólos do controlador. Isto deve-se ao fato de que os zeros alocados próximos aos pólos do estimador acabam por atenuar o efeito dos mesmos no sistema. No próximo exemplo realiza-se o posicionamento de zeros aplicados a sistemas de controle MIMO.

\subsection{Exemplo 2}

Considera-se um sistema linear invariante que representa a dinâmica de um satélite, vide (Franklin et al., 1994). Seja o sistema descrito na forma de variáveis de estado dado por:

$$
\begin{aligned}
{\left[\begin{array}{l}
\dot{x}_{1}(t) \\
\dot{x}_{2}(t) \\
\dot{x}_{3}(t) \\
\dot{x}_{4}(t)
\end{array}\right]=\left[\begin{array}{cccc}
0 & 1 & 0 & 0 \\
3 \omega_{o}^{2} & 0 & 0 & 2 \omega_{o} \\
0 & 0 & 0 & 1 \\
0 & -2 \omega_{o} & 0 & 0
\end{array}\right]\left[\begin{array}{l}
x_{1}(t) \\
x_{2}(t) \\
x_{3}(t) \\
x_{4}(t)
\end{array}\right]+\left[\begin{array}{ll}
0 & 0 \\
1 & 0 \\
0 & 1 \\
0 & 0
\end{array}\right] u(t) } \\
z(t)=\left[\begin{array}{llll}
1 & 0 & 0 & 0 \\
0 & 0 & 1 & 0
\end{array}\right]\left[\begin{array}{l}
x_{1}(t) \\
x_{2}(t) \\
x_{3}(t) \\
x_{4}(t)
\end{array}\right]
\end{aligned}
$$

sendo $x(t)$ o vetor de estados, $u(t)$ o sinal de controle, $z(t)$ a saída do sistema e $\omega_{o}$ freqüência de oscilação do sistema. Neste exemplo considera-se $\omega_{o}=1$.

Para o projeto do controlador $K$ utiliza-se a metodologia descrita no Teorema 1 e adota-se uma região para alocação dos pólos limitada de acordo a Figura 2, sendo que os parâmetros de posicionamento dos pólos são: uma circunferência de raio $\rho=20$ com centro em $(0,0)$, duas semi-retas com ângulo $\theta<85^{\circ}$ e uma região limitada a esquerda da reta vertical em $\alpha=-15$. 
Posteriormente utiliza-se a metodologia apresentada no Teorema 2 para a determinação da matriz de ganhos $L$ do estimador de estados. Neste processo adota-se uma região para o posicionamento dos pólos do estimador limitada por uma circunferência de raio $\rho_{L}=10$ com centro em $(-30,0)$. O controlador $K$ e a matriz de ganhos $L$ do estimador obtidos são apresentados a seguir:

$$
\begin{gathered}
K=\left[\begin{array}{cccc}
359,210 & 32,399 & 0,000 & 1,999 \\
0,000 & -2,000 & 356,210 & 32,399
\end{array}\right] \mathrm{e} \\
L=\left[\begin{array}{cc}
53,835 & 1,693 \\
725,456 & 19,197 \\
-1,693 & 53,835 \\
-99,197 & 722,456
\end{array}\right] .
\end{gathered}
$$

O mapeamento de pólos e zeros para este exemplo é ilustrado na Figura 7. Neste caso os zeros de malha fechada são decorrentes do uso do observador para a imposição dos pólos, e ocorrem à revelia da vontade do projetista.

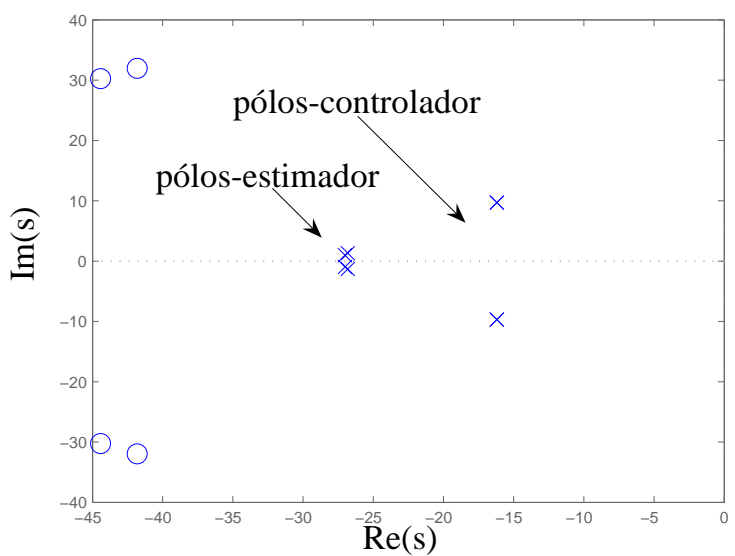

Figura 7: Mapeamento de pólos e zeros do sistema com observador e controlador.

Na Figura 8 é ilustrada a resposta do projeto sem alocação de zeros, considerando-se uma entrada do tipo degrau unitário. Observa-se na Figura 8 que o sinal de saída do sistema apresenta uma influência relativa aos pólos do estimador. A influência dos pólos do estimador no sistema pode ser verificada através da diferença entre o tempo de estabelecimento para o projeto somente com o controlador e o projeto com o controlador e o estimador. O tempo de estabelecimento da resposta do sistema MIMO de segunda ordem a uma entrada do tipo degrau unitário considerando apenas o controlador $K$ é 0,248 segundos para os dois canais de saída. Enquanto que o tempo de estabelecimento da resposta a uma entrada do tipo degrau unitário considerando-se o projeto com o esti- mador e o controlador é 0,368 segundos para os dois canais de saída.

Utiliza-se a metodologia para alocação de zeros descrita no Teorema 1 com objetivo de atenuar o efeito dos pólos do estimador no sistema. Então, aloca-se os zeros próximos aos pólos do estimador. Para o processo de alocação de zeros, adota-se a região de acordo com a Figura 2 limitada por uma circunferência de raio $\rho_{z}=4$, com centro em $(-26,0)$ do plano-s. Ainda, inclui-se 2 semi-retas com ângulo $\theta_{z}<5^{\circ}$ ao conjunto de restrições para a alocação dos zeros e um plano a esquerda da reta vertical em $\alpha_{z}=-26,1$.

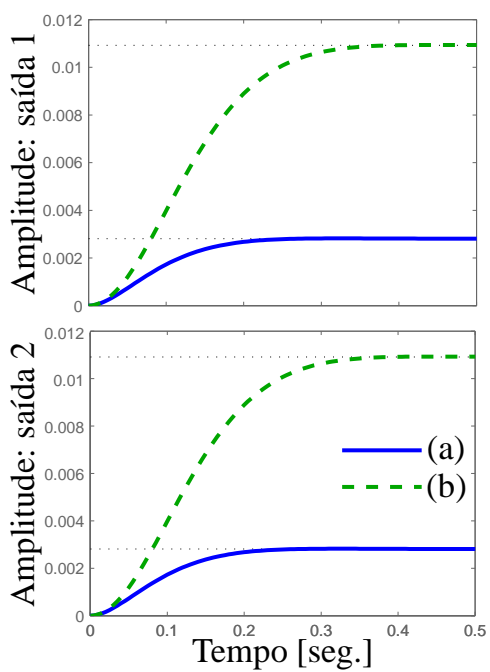

Figura 8: Resposta a entrada de um degrau unitário. (a) para o projeto somente com o controlador, (b) para o projeto com o estimador e o controlador.

Após realizar-se a alocação dos zeros utilizando o Teorema 1, utiliza-se o Teorema 7 para obter a matriz de ganhos $N$ e posteriormente obtem-se a matriz $M$. Observa-se no mapeamento de pólos e zeros ilustrado na Figura 9 que os zeros foram posicionados próximos aos pólos do estimador, e conseqüentemente diminuindo o efeito destes pólos no sistema.

Os parâmetros de alocação de zeros $M$ e $N$ obtidos são:

$$
\begin{aligned}
& M=\left[\begin{array}{cc}
-0,424 & -0,047 \\
324,734 & -7,445 \\
0.046,088 & -0,424 \\
7,380 & 324,788
\end{array}\right], \\
& N=\left[\begin{array}{cc}
336,364 & -5,064 \\
5,020 & 336,416
\end{array}\right] .
\end{aligned}
$$

Os pólos de malha fechada são: $\quad-16,199 \pm 9,684 j$; $-16,198 \pm 9,685 j$ relativos ao controlador e $-26,818 \pm$ $1,297 j ;-27,017 \pm 0,991 j$ relativos ao estimador de estados. 


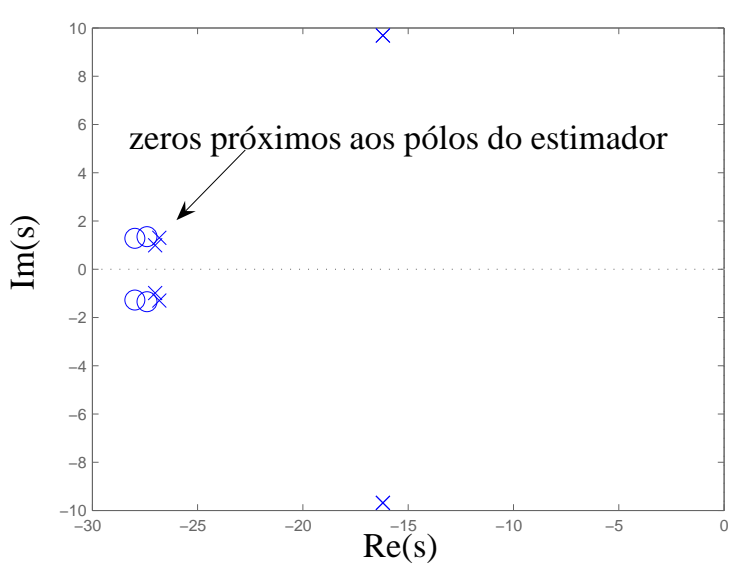

Figura 9: Mapeamento de pólos e zeros do sistema com observador, controlador e após alocação dos zeros.

Após a alocação, os zeros do sistema são: $-27,403 \pm 1,346 j$; $-27,983 \pm 1,281 j$.

Na Figura 10 é ilustrada a resposta do sistema para uma entrada do tipo degrau unitário após a alocação dos zeros. Analisando-se a Figura 10 constata-se que a resposta do sistema apresenta características relacionadas com os pólos do controlador. Este fator mostra a diminuição do efeito dos pólos do estimador ao desempenho do sistema.
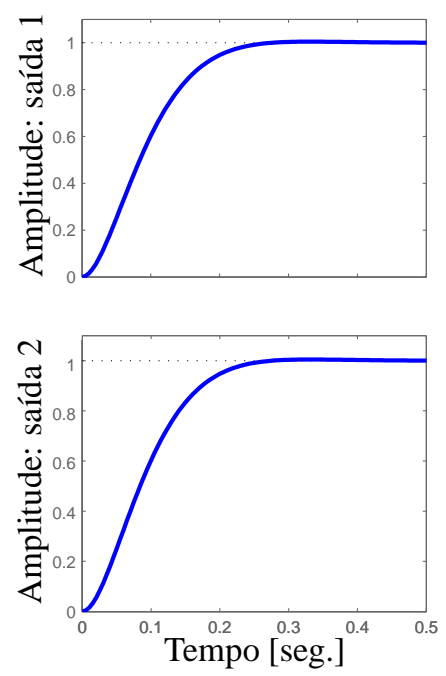

Figura 10: Resposta a entrada de um degrau unitário do sistema com observador, controlador e após alocação dos zeros.

No próximo exemplo utiliza-se a alocação de zeros para alterar a constante de erro de velocidade, $K_{v}$, de um dado sistema de controle.

\subsection{Exemplo 3}

Em (Franklin et al., 1994) é abordada uma metodologia analítica para o aumento da constante de erro de velocidade para projetos em sistemas de controle utilizando-se a alocação de zeros. Neste trabalho é proposto um projeto para o aumento de $K_{v}$, porém descrito na forma de LMIs. Considera-se o sistema de segunda ordem linear e invariante no tempo descrito na forma de variáveis de estado dado por:

$$
\begin{aligned}
{\left[\begin{array}{c}
\dot{x}_{1}(t) \\
\dot{x}_{2}(t)
\end{array}\right] } & =\left[\begin{array}{cc}
-1 & 0 \\
1 & 0
\end{array}\right]\left[\begin{array}{l}
x_{1}(t) \\
x_{2}(t)
\end{array}\right]+\left[\begin{array}{l}
1 \\
0
\end{array}\right] u(t), \\
z(t) & =\left[\begin{array}{ll}
0 & 1
\end{array}\right]\left[\begin{array}{l}
x_{1}(t) \\
x_{2}(t)
\end{array}\right],
\end{aligned}
$$

sendo $x(t)$ o vetor de estados, $u(t)$ o sinal de controle e $z(t)$ a saída do sistema.

Para o projeto do controlador $K$ utiliza-se a metodologia indicada no Teorema 1 e adota-se uma região para alocação dos pólos limitada de acordo com a Figura 2, os parâmetros de posicionamento dos pólos são: uma circunferência de raio $\rho=5$ com centro em $(0,0)$, duas semi-retas com ângulo $\theta<50^{\circ}$ e uma região limitada a esquerda da reta vertical em $\alpha=-0,5$.

Utiliza-se a metodologia apresentada no Teorema 2 para a determinação da matriz de ganhos $L$ do estimador de estados. Adota-se uma região para o posicionamento dos pólos do estimador limitada por uma circunferência de raio $\rho_{L}=5$, com centro em $(-15,0)$. O controlador $K$ e a matriz de ganhos $L$ do estimador obtidos são apresentados a seguir:

$$
K=\left[\begin{array}{ll}
2,782 & 2,672
\end{array}\right] \quad \text { e } \quad L=\left[\begin{array}{c}
152,643 \\
25,653
\end{array}\right] .
$$

Utilizando a fórmula de Truxal, vide (Franklin et al., 1994), observa-se que a constante de erro de velocidade $K_{v}$ para este exemplo é igual a 0,690 ; e deseja-se para este projeto uma aumento no valor da constante de erro de velocidade do sistema.

Os pólos de malha fechada são: $-0,940 ;-2,841$ relativos ao controlador, e $-13,326 \pm 0,832 j$ relativos ao estimador. Neste caso, os zeros decorrentes da imposição dos pólos do sistema são: $-14,717 \pm 5,980 j$.

Objetiva-se projetar $K_{v}>40$, e então, utiliza-se a alocação dos zeros. A fórmula de Truxal é dada por (Franklin et al., 1994):

$$
\frac{1}{K_{v}}=\sum \frac{1}{z_{i}}-\sum \frac{1}{p_{i}}
$$

então,

$$
\frac{1}{K_{v}}=\left(\frac{1}{z_{1}}+\frac{1}{z_{2}}\right)-\left(\frac{1}{p_{k 1}}+\frac{1}{p_{k 2}}+\frac{1}{p_{e 1}}+\frac{1}{p_{e 2}}\right),
$$


sendo, $p_{k 1}$ e $p_{k 2}$ os pólos relacionados ao controlador; e os pólos $p_{l 1}$ e $p_{l 2}$ relacionados com o estimador. Ainda, $z_{1} \mathrm{e}$ $z_{2}$ são os zeros posteriormente alocados de $r(t)$ para $u(t)$ (Figura 1).

Desta modo,

$$
\begin{aligned}
\frac{1}{K_{v}}= & \left(\frac{1}{z_{1}}+\frac{1}{z_{2}}\right)-\left(\frac{1}{-2,841}+\frac{1}{-0,940}\right. \\
& \left.+\frac{1}{-13,326+0,832 j}+\frac{1}{-13,326-0,832 j}\right), \\
\frac{1}{K_{v}}= & \left(\frac{1}{z_{1}}+\frac{1}{z_{2}}\right)+1,565
\end{aligned}
$$

considerando-se $z_{1}=z_{2}=z$ em (30), tem-se:

$$
\begin{aligned}
\frac{1}{K_{v}} & =\frac{2}{z}+1,565 ; \\
K_{v} & =\frac{z}{2+1,565 z}>40, \\
\frac{z}{2+1,565 z}-40 & >0,
\end{aligned}
$$

Analisando-se a equação (31), observa-se que o intervalo: $-1,320<z<-1,277$ satisfaz (31). Então, para a constante de erro de velocidade $K_{v}>40$ utiliza-se o Teorema 4 para alocar os zeros em uma região, de tal maneira que $-1,320<$ $z<-1,277$. Então, adota-se uma região de acordo com a Figura 2 limitada apenas por uma circunferência de raio $\rho_{z}=0,02$ com centro em $(-1,298,0)$.

Desta maneira, através da alocação dos zeros, tem-se a constante de erro de velocidade $K_{v}>40$. Os parâmetros $M$ e $N$ de alocação de zeros são descritos a seguir:

$$
M=\left[\begin{array}{c}
2471,281 \\
265,731
\end{array}\right] \text { e } N=282,648 .
$$

Os zeros de $r(t)$ para $u(t)$ alocados são: $-1,291 \mathrm{e}-1,305$, observa-se que os zeros alocados estão dentro da faixa especificada em projeto para que a constante de erro de velocidade $K_{v}>40$.

A resposta a uma entrada do tipo rampa é ilustrada na Figura 11. Observa-se que o erro de regime para uma entrada do tipo rampa diminuiu após a elevação da constante de erro de velocidade, $K_{v}$, utilizando a alocação de zeros proposta no Teorema 4.

Neste exemplo utilizou-se a alocação dos zeros para aumentar a constante de erro de velocidade de um dado sistema, e verificou-se que, alocando-se os zeros de maneira adequada pode-se especificar $K_{v}$ de acordo com a necessidade do projetista. Esta metodologia descrita na forma de LMIs possibilitará a abordagem de sistemas incertos, que ainda é tema de

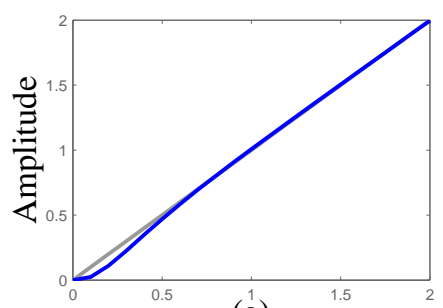

(a)

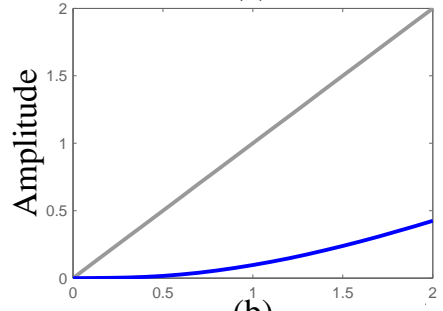

Tempo [seg.]

Figura 11: Resposta a entrada do tipo rampa $r(t)=t$ : (a) com alocação dos zeros, (b) sem alocação dos zeros.

pesquisa dos autores. Uma vantagem é que pode-se abordar projetos de sistema de controle com alocação de zeros para o caso MIMO que não existe na literatura.

\section{CONCLUSÃO}

Neste trabalho apresentou-se uma metodologia sistemática de alocação de zeros via LMIs aplicada em sistemas de controle. A região proposta para alocação dos zeros é mostrada na Figura 2, e é limitada por uma circunferência de raio $\rho_{z}$ com centro em $-q_{z}$, por uma reta vertical em $-\alpha_{z}$ e por duas semi-retas de ângulo $\theta_{z} \mathrm{e}-\theta_{z}$.

Utilizou-se o posicionamento dos zeros para diminuir ou até mesmo anular o efeito de pólos que não sejam de interesse do projetista. Ainda, corrigiu-se o erro de regime para uma entrada do tipo degrau unitário através de uma escolha adequada do ganho $N$ em função da matriz $M$, o que pode ser visualizado no primeiro exemplo.

Outro tópico abordado foi a alteração da constante de erro de velocidade $K_{v}$ de um dado sistema através do posicionamento dos zeros. No segundo exemplo numérico aumentouse esta constante para um valor especificado em projeto. Enfim, o posicionamento dos zeros é uma ferramenta adicional que tem o objetivo ampliar as opções para projetos em sistema de controle.

O projeto de posicionamento dos zeros é descrito na forma de LMIs, que quando factível pode ser facilmente solucionado através de algoritmos de convergência polinomial disponíveis na literatura ((Gahinet et al., 1995), (de Oliveira et al., 1997)). Pelo conhecimento dos autores, a alocação de zeros em uma região via LMI é original e inédita. Como o 
projeto é descrito na forma de LMIs, pode-se incluir incertezas paramétricas, o que torna a metodologia proposta robusta (Andrea et al., 2004). Isto é objeto de pesquisa por partes dos autores.

\section{REFERÊNCIAS}

Andrea, C. Q., Assunção, E. and Teixeira, M. C. M. (2004). Controle Ótimo $\mathcal{H}_{2}$ e $\mathcal{H}_{\infty}$ com Modificação de Zeros para o Problema de Rastreamento em Sistemas Lineares Incertos, $3^{\circ}$ Congresso Temático de Dinâmica e Controle da SBMAC, Ilha Solteira, SP, Brasil .

Assunção, E. and Peres, P. L. D. (1999). A Global Optimization Approach for the $\mathcal{H}_{2}$-norm Model Reduction Problem, Proceedings of the 38th IEEE Conference on Decision and Control, Phoenix, AZ, USA, pp. 18571862.

Assunção, E., Andrea, C. Q. and Teixeira, M. C. M. (2004). Controle Ótimo $\mathcal{H}_{2}$ e $\mathcal{H}_{\infty}$ com Modificação de Zeros para o Problema de Rastreamento Usando LMIs, SBA Controle \& Automação. 15(4): 413-422.

Boyd, S., El Ghaoui, L., Feron, E. and Balakrishnan, V. (1994). Linear Matrix Inequalities in Systems and Control Theory, SIAM Studies in Applied Mathematics, USA.

Chilali, M. and Gahinet, P. (1996). $\mathcal{H}_{\infty}$ Design with Pole Placement Constraints: An LMI Approach, IEEE Transactions on Automatic Control 41(3): 358-367.

da Silva, S., Lopes J., V. and Assunção, E. (2004). Robust Control of Truss Structure Using Linear Matrix Inequalities, 22-th International Modal Analysis Conference IMAC, Dearborn, Michigan, USA.

de Oliveira, M. C., Farias, D. P. and Geromel, J. C. (1997). LMISol, User's Guide, UNICAMP, CampinasSP, Brasil. http://www.dt.fee.unicamp.br/ mauricio/software.html.

Dorf, R. C.and Bishop, R. H. (2001). Sistemas de Controle Modernos, oitava edição edn, LTC - Livros Técnicos e Científicos Editora S.A., Rio de Janeiro, Brasil.

Franklin, G. F., Powell, J. D. and Emami-Naeini, A. (1994). Feedback Control of Dynamic Systems, Addison Wesley, USA.

Gahinet, P., Nemirovski, A., Laub, A. J. and Chilali, M. (1995). LMI Control Toolbox User's Guide, The Mathworks Inc., USA.

Hauksdóttir, A. S. (2000). Optimal Zeros for Model Reduction of Continuous-Time System, $39^{\text {th }}$ IEEE Conference on Decision and Control pp. 1889-1893.
Herjólfsson, G., Ævarsson, B., Hauksdóttir, A. S. and Sigurðsson, S. P. (2005). Zero Optimized Tracking for Linear Continuous-Time Systems, In Proceedings of the American Control Conference, Portland, OR, EUA, pp. 1208-1213.

Lee, S., Meekov, S. M. and Runolfsson, T. (1987). Vibrational Feedback Control: Zeros Placement Capabilities, IEEE Transactions on Automatic Control AC32(7): 604-611.

Moore, K. L. and Bhattacharyya, S. P. (1990). A Technique for Choosing Zero Locations for Minimal Overshoot, IEEE Transactions on Automatic Control 35(5): 577580.

Nesterov, Y. and Nemirovsky, A. (1994). Interior-Point Polynomial Algorithms in Convex Programming, SIAM Studies in Applied Mathematics, USA.

Ogata, K. (1997). Modern Control Engineering, PrenticeHall, 3rd. ed., New Jersey, USA.

Palhares, R. M., Hell, M. B., Durães, L. M., Ribeiro Neto, J. L., Teixeira, M. C. M. and Assunção, E. (2003). Robust $\mathcal{H}_{\infty}$ Filtering for a Class of State-delayed Nonlinear Systems in an LMI Setting, International Journal Of Computer Research 12(1): 115-22.

Saeks, R. and Murray, J. (1981). Feedback System Design: The Tracking and Disturbance Rejection Problems, IEEE Transactions on Automatic Control AC26(1): 203-217.

Teixeira, M. C. M., Assunção, E. and Avellar, R. G. (2003). On Relaxed LMI-Based Designs for Fuzzy Regulators and Fuzzy Observers, IEEE Transactions on Fuzzy Systems 11(5): 613-623.

Teixeira, M. C. M., Assunção, E. and Pietrobom, H. C. (2001). On Relaxed LMI-Based Design Fuzzy, Proceedings of the 16th European Control Conference, Porto, Portugal, pp. 120-125.

Teixeira, M. C. M., Covacic, M., Assunção, E. and Lordelo, A. D. (2002). Design of SPR Systems and Output Variable Structure Controllers Based on LMI, 7th IEEE International Workshop on Variable Structure Systems, Vol. 1, Sarajevo, Bosnia, pp. 133-144.

Teixeira, M. C. M. and Zak, S. H. (1999). Stabilizing Controller Design for Uncertain Nonlinear Systems Using Fuzzy Models, IEEE Transactions on Fuzzy Systems 7(2): 133-142.

Tu, M. C. and Lin, C. M. (1992). Robust Stabilisation of Multivariable Feedback Systems with Desired Performance Requirement, IEE Proceedings 139(3): 259266. 